\title{
OBITUARY
}

\section{ANIBAL RUIZ MORENO, 1907-60}

Professor Anibal Ruiz Moreno, M.D., professor of medical history in the medical faculty of the university of Buenos Aires and one of the leading rheumatologists in the Argentine, died suddenly on December 22, 1960, at the age of 54, while lecturing to his students.

He had worked in the department of medical history for 25 years, and had been director of the rheumatology department for over 20 years.

He was founder and president of the Argentine League against Rheumatism, president of the PanAmerican League, and president-elect of the International League.

In 1938 he founded the periodical Archivos Argentinos de Reumatologia, which is now in its 24th year, and as well as editing this journal, and publishing numerous books on the historical aspects of medicine and rheumatology, he contributed very many articles to medical journals throughout the world, as reference to the Annals Abstracts Section will quickly show.

His many friends amongst his fellow rheumatologists missed his presence and that of his gracious wife, Luisa Allende de Ruiz Moreno, at the Tenth International Congress in Rome in September, when he was to have been elected president for the ensuing four years.

Dr. François Françon writes: Avec le professeur Anibal Ruiz Moreno disparaît une grande figure de la rhumatologie mondiale: il a été véritablement un des grands fondateurs de notre discipline en la République Argentine.

Il a été frappé le 22 décembre 1960, sur son "champ de bataille", comme le disait Corvisart en parlant de la mort de Bichat, alors qu'il présidait un séminaire dans sa chaire d'Histoire de la Médecine.

Ruiz Moreno était né à Cordoba (Argentine) le 31 mai 1907, et il avait fait ses études médicales à $\overrightarrow{0}$ la Faculté de Buenos-Ayres où il avait soutenu, en 1932, une thèse remarquée sur "les relations entre les secrétions internes et externes du pancréas". Il s'était ensuite consacré à l'Histoire de la Médecine et à la Rhumatologie.

A ce dernier titre il avait créé la Ligue Argentine contre le Rhumatisme, qui est devenue maintenant la Société Argentine de Rhumatologie: elle m'a accordé la faveur d'être élu membre d'honneur. Il avait été fondateur, puis Vice-Président et enfin Président de la Ligue Panaméricaine contre le Rhumatisme: il avait été élu Président-désigné de la Ligue Internationale contre le Rhumatisme pour la période 1961-1965, et, en cette qualité, il devait $\stackrel{\Phi}{-}$ assurer la Présidence du Congrès de Rome les $3-\widehat{\delta} \overrightarrow{0}$ septembre 1961; un sort cruel lui a enlevé ce couronnement mérité de ses efforts et de toute ung vie de labeur.

Il a fondé le Centre Antirhumatismal de BuenosAyres et les Archivos Argentinos de Reumatologia, organe trimestriel, qui atteint sa $24^{\circ}$ année d'existence. Il a écrit quatre livres et plus de centcinquante articles sur la rhumatologie; il a dirigé de nombreux cours et il a été Rapporteur à maints congrès nationaux et internationaux: il était membre d'honneur de dix-sept Sociétés étrangères de Rhumatologie.

Son activité n'a pas été moindre dans le champ national et international d'Histoire de la Médecine dont il était professeur titulaire depuis 1947.

Nous avions eu la bonne fortune de le rencontrer à différents congrès; en même temps qu'un grand savant, il était un hidalgo accompli et il incarnait merveilleusement le type physique, les qualités et les aspirations de la belle race argentine.

Que sa veuve et sa famille veuillent bien trouver $N$ ici l'expression de nos condoléances émues et de $N$ notre vive gratitude. 